\section{Characterization and Identification of Indigenous Olive Germplasm from Cyprus Using Morphological and Simple Sequence Repeat Markers}

\author{
Maria G. Emmanouilidou ${ }^{1,2}$ and Marios C. Kyriacou \\ Agricultural Research Institute, P.O. Box 22016, 1516, Nicosia, Cyprus \\ Isabel Trujillo ${ }^{2}$ \\ Department of Agronomy, Campus Universitario de Rabanales, Edf. \\ Celestino Mutis, Ctra. Madrid-Cádiz, Km. 396, 14014, Córdoba, Spain
}

Additional index words. Olea europaea, microsatellites, ex situ conservation, clonal selection, molecular variants, genetic diversity

\begin{abstract}
Implementation of the recently developed Cyprus National Register of Commercial Varieties mandates proper cataloguing of the material conserved in the ex situ Olive Collection at the Agricultural Research Institute of Cyprus. A total of 125 trees belonging to 32 accessions were analyzed using 11 morphological endocarp traits and 14 microsatellite [simple sequence repeat (SSR)] markers. The SSR variability allowed segregation of 16 genotypes among accessions, which were clustered into three main groups based on genetic similarity. Similarity indexes among groups ranged from 0.63 to 0.65 and within groups were $\geq 0.9$. Lower morphological variability was detected among accessions, which conformed to three morphological profiles; moreover, the three morphological profiles corresponded to the three groups of genetic similarity obtained by SSR markers. The identification, based on the unique combination of SSR genotypes and endocarp morphologies, revealed the presence of three cultivars and 15 molecular variants that presented limited molecular differences but morphological profiles identical to their catalogued cultivars. Two cultivars, 'Ladoelia' and 'Kato Drys', demonstrated molecular variation. The current study underpins the efficient management of olive germplasm collections based on combined molecular and phenotypic characterization of their accessions. The complete cataloguing of olive germplasm ensures the correct distribution of identified and authenticated material across national or international institutions.
\end{abstract}

The olive (Olea europaea L.) is among the most ancient crops of the Mediterranean basin (Zohary and Spiegel-Roy, 1975). In Cyprus, as in the entire eastern Mediterranean region, olive trees coexisted with humans in almost all Neolithic settlements (6000-3000 BCE); however, the initiation of olive culture dates to the second millennium BCE (Hadjisavvas, 1992). The olive crop is characterized by the existence of several clonally propagated traditional cultivars, which emerged through the empirical and local selection of exceptional trees (Trujillo et al., 2014). Subsequently, the spread of olive culture westwards across the Mediterranean basin by successive centers of diffusion led to complex genetic relations among cultivars (Besnard et al., 2013; Rallo, 2005; Trujillo et al., 2014).

Received for publication 10 May 2018. Accepted for publication 23 July 2018 .

This research was held during the sabbatical stay of Mrs. Maria Emmanouilidou in Spain as a fellow of International Olive Council and in the context of her research affiliation with the Agricultural Research Institute of Cyprus.

${ }^{1}$ First author.

${ }^{2}$ Corresponding authors. E-mail: maria.emmanouelidou@, ari.gov.cy or ag2trnai@uco.es.
At present, traditional olive groves in Cyprus mainly are established with very old varieties, which yet remain unidentified due to the scarcity of systematic surveys and characterization studies on local olive germplasm. The traditional system of olive culture coexists with modern olive groves established with reputable foreign varieties introduced on the island since the late 1970s. Marked examples of such varieties, regarded as select oil varieties, include the 'Koroneiki', 'Picual', and 'Moraiolo', introduced from Greece, Spain, and Italy, respectively. Among table olive varieties most common are 'Kalamon' and 'Amfissis' from Greece and 'Manzanilla de Sevilla' from Spain. The lack of systematic information on local olive germplasm curbs the dynamic exploitation of the local ancient genetic material and facilitates the preferential use of profusely characterized foreign varieties.

Gene banks ensure the long-term ex situ conservation of valuable genetic resources and command a vital role in the identification process of olive varieties. Their activity also contributes significantly to the control of genetic erosion of local olive germplasm, which in recent years poses a heightened risk as a result of the intensification of olive culture. The principle systematic survey conducted on indigenous olive germplasm from Cyprus focused solely on the clonal selection of the cv. Ladoelia, considered the main local variety (Gregoriou, 1996, 1999). The material collected in this study was conserved in the Olive Collection of the Agricultural Research Institute of Cyprus (OCARICY). This collection was partially characterized using random amplified polymorphic DNA markers (Banilas et al., 2003) and broad agronomic characters such as trunk cross-sectional area, yield per tree, fruit weight, fruit and endocarp length and diameter, fruit flesh percentage, and oil content (Gregoriou, 1996, 1999). The results obtained in these studies revealed high random amplified polymorphic DNA polymorphism as well as agronomic differences, identified mainly in respect to fruit weight and oil content.

On the basis of the guidelines set out by the European Union on varietal olive certification, Cyprus has launched the development of the National Register of Commercial Varieties. The implementation of the Register requires a systematic characterization study for the material of OCARICY as well as the application of more current methodological protocols for varietal identification. In this regard, a protocol recently has been established based on the integrated use of morphological and molecular markers (microsatellites) to facilitate the identification of the varieties present in olive gene banks. This protocol has allowed identification of the World Olive Germplasm Bank of Cordoba (WOGBC) and established a broad base of molecular data that can be exploited in crossing data between diverse olive gene banks (Trujillo et al., 2014). Of several systems of morphological characterization applied on olive, the most optimal is the systematic use of pomological patterns, which include a large number of traits related to tree, leaf, fruit, and endocarp (Barranco et al., 2000, 2005). This protocol was adopted and referenced by the International Union for the Protection of New Varieties of Plants in 2011. Traits related to the endocarp stand out for their major discriminative capacity, limited fluctuation, and ease of conservation (Barranco et al., 2005; Trujillo et al., 2006). For these reasons, the description of the endocarp frequently has been used to catalogue olive cultivars (Barranco et al., 2000, 2005; Fendri et al., 2010; D'Imperio et al., 2011) and large collections of olive germplasm (Trujillo et al., 2014).

The polymerase chain reaction (PCR)SSR technique has been used widely in olive varietal characterization, as it consists of a relatively simple methodology that permits an easy interpretation of results (Fendri et al., 2010; Rafalski and Tingey, 1993). Actually, the microsatellite markers (SSRs) are considered as leading markers for conducting varietal characterization and identification studies on olive (Anestiadou et al., 2017; Baldoni et al., 2009; Carriero et al., 2002; 
Cipriani et al., 2002; De La Rosa et al., 2002; Díez et al., 2011, 2012; D'Imperio et al., 2011; Fendri et al., 2010; Haouane et al., 2011; Rallo et al., 2000; Sefc et al., 2000; Trujillo et al., 2014). Their discriminative capacity, consistency, codominant nature, easy interpretation, and low cost account for their success.

The denomination of olive cultivars is usually a process synchronous to their diffusion. Accordingly, it may be based on outstanding morphological traits, on intended use, on geographical origin of the propagated material, or on other characteristics (Rallo, 2005; Trujillo et al., 2014). As a result of the large number of varieties and the fact that their denomination is based mainly on generic criteria and local origin, the existence of synonymy (different names for the same cultivar) and homonymy (same name for different cultivars) is very common among and within olive-growing countries (Barranco et al., 2000). It is estimated that there are more than 2000 olive varieties worldwide (Lavee, 1994), whereas 1200 autochthonous varieties with more than 3000 denominations have been documented (Bartolini et al., 1998). The crossing of characterization data (morphological and molecular) for olive varieties under identification with authenticated varieties of reference collections is the final step in the process of cultivar identification, capable of demonstrating the existence of synonymies. In Cyprus, it is believed that the local olive genetic material belongs entirely to one variety, although the results from the studies previously performed on olive by Gregoriou $(1996,1999)$ and Banilas et al. (2003) revealed in some cases a greater than expected morphological and genetic variability in the material derived from clonal selection.

The main objective of the present study has been the characterization and identification of the genetic material included in the OCARICY collection using up-to-date molecular and morphological protocols optimized for olive. The current study facilitates the efficient management of olive germplasm collections based on combined molecular and phenotypic characterization of their accessions and highlights the promotion of agronomically valuable germplasm. In respect to the Cyprus indigenous germplasm, the current study lays the foundation for further enrichment of the OCARICY ex situ collection with additional olive genetic material currently preserved in situ under the threat of genetic erosion (Anestiadou et al., 2017). Moreover, the present work contributes toward the advancement of characterization studies on indigenous olive genetic material, as well as toward the furthering of transnational relations between gene banks of similar scope.

\section{Materials and Methods}

Plant material. Samples of 32 accessions of cultivated olive (Olea europaea L.) from the ex situ OCARICY were analyzed
(Table 1). The OCARICY material was previously collected in a systematic survey of clonal material of the variety Ladoelia performed by Gregoriou (1996, 1999). Each accession was given a unique identifier code composed by the letters ARICY plus four digits corresponding to the order of registration of the accession to the collection (e.g., ARICY0001). Aside from identifier codes, during the survey study names were assigned to accessions circumstantially that usually designated the geographical region of origin; for example, the accession referenced as Kato Drys (ARICY0008) had been localized at the village of Kato Drys in the district of Larnaca (Table 1).

Preliminary morphological characterization was carried out on all 125 trees, which comprised the 32 accessions of OCARICY, provided they exhibited satisfactory production load and typical fruit morphology. Molecular characterization of one tree per accession was subsequently performed on all accessions with the exception of Arediou 3, Kato Drys 2, Kiti, Klirou 2, Lythrodontas, and Meniko. In the latter six accessions, minor variability was observed in the size and shape of fruits during the initial morphological characterization, which prompted more extensive sampling (of two or three trees) for molecular characterization (Table 1). All the accessions examined were self-rooted (nongrafted). Further to the accession identifier code, each of the trees analyzed also was assigned an individual identifier code according to its position within the collection (Table 2; Supplemental Table 1).

Morphological analysis. We evaluated 11 traits of the endocarp described by Barranco et al. $(2000,2005)$ and adopted by International Union for the Protection of New Varieties of Plants (2011): weight, length/ width ratio, symmetry in position $\mathrm{A}$, symmetry in position $\mathrm{B}$, position of the maximum transversal diameter $B$, shape of apex in position $\mathrm{A}$, shape of base in position $\mathrm{A}$, rugosity of surface, number of grooves, distribution of grooves on basal end, and presence of mucron (Table 3; Supplemental Table 1). Morphological characterization was carried out on representative samples of 50 endocarps per tree for at least 3 years. The morphological profile of each sample was designated a numerical code configured by the combined expression of the 11 endocarp traits evaluated (Table 3; Supplemental Table 1).

DNA extraction and PCR-SSR analysis. Total genomic DNA was isolated from fresh, young leaves following a CTAB protocol proposed by Murray and Thompson (1980) and further modified by De La Rosa et al. (2002). A set of 14 microsatellite markers were analyzed: ssrOeUA-DCA3, ssrOeUADCA9, ssrOeUA-DCA11, ssrOeUA-DCA15, ssrOeUA-DCA16, ssrOeUA-DCA18 (Sefc et al., 2000), GAPU-59, 71B, 101, 103A (Carriero et al., 2002), UDO99-011, 019, 024, and 043 (Cipriani et al., 2002) (Table 4). All of them have been described as very efficient for olive cultivar identification studies (Baldoni et al., 2009; Trujillo et al., 2014). Differences of $1 \mathrm{bp}$ between alleles were checked by reamplification to establish whether a coding error had occurred. In the case of six accessions, referred to in the previous Plant Material section, replicate trees were compared. In cases of mismatch, replicates were analyzed again using newly collected plant material. Such cases of mismatch were considered correct SSR profiles if differences were confirmed in the repeat amplification products.

The microsatellite amplification was conducted on a final volume of $20 \mu \mathrm{L}$, containing 2 ng of genomic DNA, 1× supplies PCR buffer (Biotools, Madrid, Spain), $200 \mu \mathrm{M}$ each dNTP (Roche, Indianapolis, IN), $1.5 \mathrm{~mm}$ $\mathrm{MgCl}_{2}, 0.25$ units of Taq DNA polymerase (Biotools), and $0.2 \mu \mathrm{M}$ forward (fluorescently labeled) and reverse primers. The PCRs were carried out on a thermal cycler (Perkin Elmer 9600; Applied Biosystems, Foster City, CA) using the following program: denaturation at $94{ }^{\circ} \mathrm{C}$ for $5 \mathrm{~min}, 35$ cycles of $94{ }^{\circ} \mathrm{C}$ for $20 \mathrm{~s}$, 50 to $59^{\circ} \mathrm{C}$ for $30 \mathrm{~s}, 72^{\circ} \mathrm{C}$ for $30 \mathrm{~s}$, and a final extension at $72{ }^{\circ} \mathrm{C}$ for $8 \mathrm{~min}$. The detection of amplification products was carried out with an automatic sequencer, the ABI $3130 \mathrm{Ge}$ netic Analyzer (Applied 181 Biosystems/ Hitachi, Waltham, MA) using the internal standard GeneScan 400 HD-Rox. The cultivars Frantoio and Picual were used as controls in all runs.

Molecular data analysis. The allele profiles were sized in base pairs and characterized using GeneScan 3.7 (Applied Biosystems). A numeric code was assigned to different SSR profiles defined among trees analyzed (Tables 1 and 2). In addition, for each SSR locus the following parameters were calculated using software program Power Marker V3.23 (Liu and Muse, 2005): average number of alleles; number of unique alleles, presented only by a single genotype; observed heterozygosity; expected heterozygosity; and polymorphism information content (Botstein et al., 1980). The identity probability (PI) (Paetkau and Strobeck, 1994) for each locus and for the whole SSR set (accumulated PI) was calculated using the software Gimlet v1.3.3 (Valière, 2002).

The evaluation of the genetic relationships among the different genotypes was held by the construction of a matrix containing only the different SSR profiles, where the amplified alleles scored as present (1) or absent (0). Finally, this matrix was used to perform a cluster analysis based on the unweighted pair group method with arithmetic mean algorithm using Dice's similarity index (Dice, 1945) on NTSYS-PC v2.02 software (Rohlf, 1998). The correlation coefficient between the similarity matrix and the cophenetic values matrix was computed to test the goodness of fit for the cluster analysis.

Identification and naming of cultivars. The discrimination among different cultivars was conducted via the pairwise comparison of their SSR and morphological profiles. For the verification of the distinctness or similarity of 
Table 1. Identification of the 32 OCARICY accessions analyzed: collection code, accession name, origin, number of trees analyzed by morphological traits (Morph) and/or SSR markers (SSRs), their morphological and SSR codes, and cultivar name. ${ }^{z}$

\begin{tabular}{|c|c|c|c|c|c|c|c|c|}
\hline \multirow[b]{2}{*}{ Accession code } & \multirow[b]{2}{*}{ Accession name } & \multirow[b]{2}{*}{ Origin } & \multicolumn{2}{|c|}{ Number of trees analyzed } & \multicolumn{3}{|c|}{ Identification } & \multirow[b]{2}{*}{ Comment } \\
\hline & & & Morph & SSRs & Morph code & SSR code & Cultivar name & \\
\hline ARICY0014 & Alethriko & Larnaca & 4 & 1 & 2 & 12 & Ladoelia & MV \\
\hline ARICY0006 & Analiontas 2 & Nicosia & 5 & 1 & 2 & 14 & Ladoelia & MV \\
\hline ARICY0017 & Arediou 1 & Nicosia & 3 & 1 & 2 & 5 & Ladoelia & \\
\hline ARICY0018 & Arediou 2 & Nicosia & 7 & 1 & 2 & 5 & Ladoelia & \\
\hline ARICY0010 & Evrichou 1 & Nicosia & 4 & 1 & 2 & 8 & Ladoelia & MV \\
\hline ARICY0011 & Evrichou 2 & Nicosia & 6 & 1 & 2 & 5 & Ladoelia & \\
\hline ARICY0012 & Evrichou 3 & Nicosia & 4 & 1 & 2 & 13 & Ladoelia & MV \\
\hline ARICY0013 & Flasou & Nicosia & 5 & 1 & 2 & 5 & Ladoelia & \\
\hline ARICY0008 & Kato Drys & Larnaca & 3 & 1 & 1 & 3 & Kato Drys & MV \\
\hline ARICY0026 & Kato Drys 1 & Larnaca & 5 & 1 & 1 & 4 & Kato Drys & MV \\
\hline ARICY0027 & Kato Drys 2 & Larnaca & 5 & 2 & 2 & 5 & Ladoelia & \\
\hline ARICY0028 & Lefkara & Larnaca & 6 & 1 & 2 & 5 & Ladoelia & \\
\hline ARICY0023 & Lefkara 1 & Larnaca & 2 & 1 & 2 & 5 & Ladoelia & \\
\hline ARICY0029 & Lefkosia & Nicosia & 7 & 1 & 2 & 6 & Ladoelia & MV \\
\hline ARICY0004 & Lythrodontas & Nicosia & 4 & 2 & 2 & 5 & Ladoelia & \\
\hline ARICY0032 & Mazotos & Larnaca & $\mathrm{na}^{\mathrm{y}}$ & 1 & 2 & 5 & Ladoelia & \\
\hline ARICY0003 & Meniko & Nicosia & 2 & 2 & 2 & 11 & Ladoelia & MV \\
\hline ARICY0031 & Meniko 2 & Nicosia & 1 & 1 & 2 & 10 & Ladoelia & MV \\
\hline ARICY0002 & Paliometocho & Nicosia & 5 & 1 & 2 & 5 & Ladoelia & \\
\hline ARICY0019 & Peristerona 1 & Nicosia & 3 & 1 & 2 & 7 & Ladoelia & MV \\
\hline ARICY0020 & Peristerona 2 & Nicosia & 4 & 1 & 2 & 12 & Ladoelia & MV \\
\hline - & Unknown & - & 1 & 1 & 1 & 1 & Kato Drys & MV \\
\hline Total & & & 125 & 39 & & & & \\
\hline
\end{tabular}

${ }^{\mathrm{z}}$ Accessions marked as MV denote molecular variants, i.e., different genotypes with a similarity index $>0.9$ that correspond to the same morphological description code of the corresponding cultivar.

${ }^{\mathrm{y}}$ No sample available.

OCARICY = Olive Collection of the Agricultural Research Institute of Cyprus; SSR = simple sequence repeat.

the defined cultivars in this study, from other potentially related accessions, their profiles were compared with the SSR and morphological profiles obtained from the varietal identification of the WOGBC, Spain (Trujillo et al., 2014). This is one of the world's largest collections of olive germplasm, which currently includes profiles belonging to 500 olive accessions from 21 countries.

To assign correct varietal names to the 32 accessions evaluated in this work, we used the same criteria described in Barranco et al. $(2000,2005)$ and Trujillo et al. (2014) and according to the International Code of Nomenclature for Cultivated Plants (Brickell et al., 2009) as follows:

Cursive letter: Initial name of the accession before its identification.

Name between quotation marks: Name of a cultivar after the processes of molecular and morphological identification.

\section{Results and discussion}

Morphological and molecular variability evidenced in OCARICY. The 11 endocarp traits examined were presented morphological variability, with the exception of the characteristic presence of mucron, evidenced throughout all endocarps evaluated. The characterization of 125 samples of endocarps allowed the definition of three phenotypes or morphological profiles (Table 3; Supplemental Table 1). Phenotypes 1 and 2 grouped four and 26 accessions, respectively, whereas phenotype 3 included invariably all trees of accession Korakou. Pairwise morphological differences among phenotypes ranged from nine characteristics (phenotypes 1 vs. 2) to six characteristics (phenotypes 2 vs. 3; Table 3; Fig. 1). Fendri et al. (2010), by the application of the same 11 endocarp traits for the characterization and identification of Tunisian olive germplasm, also revealed clear differences in all the endocarp characters analyzed, between individuals identified as independent genotypes.

Absence of morphological variability between trees was observed in all accessions, including the accessions Arediou 3, Kato Drys 2, Kiti, Klirou 2, Lythrodontas, and Meniko, for which preliminary evidence had suggested limited morphological variability among accession tress and prompted extensive molecular characterization.

Regarding the SSR variability, 47 alleles were amplified with the 14 SSR loci analyzed. The number of alleles per SSR ranged from one (ssrOeUA-DCA15 and UDO99-019) to six (ssrOeUA-DCA16 and UDO99-43), with an average of 3.4 alleles per locus (Table 4). The level of polymorphism obtained was similar to that obtained in previous studies carried out on a limited number of genotypes (Charafi et al., 2008; D'Imperio et al., 2011; Muzzalupo et al., 2010) whereas higher polymorphism was obtained by SSRs markers on a recent study of centennial olive trees in which some cv. Ladoelia accessions and foreign cultivars were included (Anestiadou et al., 2017). The mean observed heterozygosity $(0.8)$ was higher than the expected heterozygosity $(0.48)$, with values ranging from 0.08 to 1 and from 0.08 to 0.71 , respectively (Table 4). The highest polymorphism information content presented by UDO99043 is in agreement with the ranking of markers used in previous olive studies. Also, the order of information level of the 14 SSRs analyzed was similar to the order obtained in other studies for same set of SSRs (Fendri et al., 2010; Trujillo et al., 2014). The total cumulative PI value for this set of markers was $6.44 \times 10^{-7}$, which shows the high discrimination power of the SSR set used.

In fact, 16 allelic profiles or genotypes were amplified among 39 trees analyzed with the 12 polymorphic SSR loci. It should be 







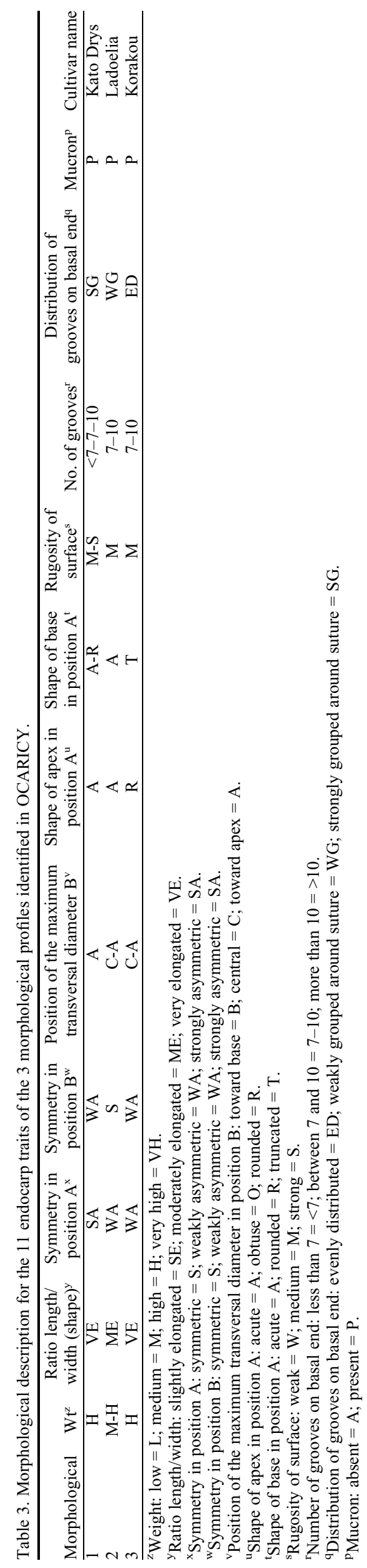

Table 4. Diversity parameters for the 14 SSR markers used.

\begin{tabular}{lcccccccc}
\hline Locus & Size range $(\mathrm{bp})$ & $\mathrm{Na}$ & $\mathrm{Nu}$ & $\mathrm{He}$ & $\mathrm{Ho}$ & $\mathrm{PIC}$ & $\mathrm{PI}$ & $\mathrm{MV}$ \\
\hline ssrOeUA-DCA3 & $243-249$ & 3 & 1 & 0.52 & 1 & 0.395 & 0.348 & Yes \\
ssrOeUA-DCA9 & $160-202$ & 5 & 2 & 0.6 & 1 & 0.509 & 0.236 & Yes \\
ssrOeUA-DCA11 & $146-160$ & 4 & 1 & 0.59 & 1 & 0.492 & 0.252 & Yes \\
ssrOeUA-DCA15 & 243 & 1 & - & - & - & - & - & No \\
ssrOeUA-DCA16 & $122-228$ & 6 & 3 & 0.58 & 1 & 0.484 & 0.259 & Yes \\
ssrOeUA-DCA18 & $166-174$ & 3 & 1 & 0.08 & 0.08 & 0.077 & 0.841 & Yes \\
UDO99-011 & $114-119$ & 2 & - & 0.5 & 0.86 & 0.37 & 0.373 & No \\
UDO99-019 & 129 & 1 & - & - & - & - & - & No \\
UDO99-024 & $164-185$ & 3 & - & 0.58 & 1 & 0.476 & 0.268 & No \\
UDO99-043 & $202-221$ & 6 & 2 & 0.71 & 0.81 & 0.643 & 0.132 & Yes \\
GAPU59 & $206-216$ & 3 & 1 & 0.51 & 0.86 & 0.389 & 0.352 & No \\
GAPU71B & $121-141$ & 3 & - & 0.58 & 1 & 0.475 & 0.268 & No \\
GAPU101 & $189-197$ & 2 & - & 0.5 & 0.86 & 0.37 & 0.373 & No \\
GAPU103A & $137-171$ & 5 & 3 & 0.55 & 1 & 0.433 & 0.309 & Yes \\
Mean & & 3.4 & 1 & 0.48 & 0.8 & 0.391 & & \\
Total & & 47 & 14 & & & & $6.44 \times 10^{-7}$ & \\
\hline
\end{tabular}

$\mathrm{bp}=$ base pair; $\mathrm{Na}=$ number of alleles; $\mathrm{Nu}=$ number of unique alleles; $\mathrm{Ho}=$ observed heterozygosity; $\mathrm{He}=$ expected heterozygosity; PIC = polymorphic information content; $\mathrm{PI}=$ probability of identity; $\mathrm{MV}=$ whether the SSR was involved in the description of molecular variants.

noted that the same number of genotypes could be defined using a set of eight SSR loci (UDO99-043, ssrOeUA-DCA9, ssrOeUADCA11， ssrOeUA-DCA16，UDO99-024, GAPU71B, GAPU103, and ssrOeUA-DCA3). Each genotype was coded with an ordinal number from 1 to 16 . (Table 2). The distribution of the accessions per genotype was as follows: a) 11 accessions showed unique SSR profiles (not duplicated in any other accession) and b) 21 accessions shared SSR profiles with other accessions resulting in the identification of the five remaining SSR profiles (Tables 1 and 2). No molecular variability was found within the accessions where more than one tree was analyzed (Table 1), and the minor morphological deviations recorded in preliminary observations performed before the present study (data not presented) may be attributed to agronomic and climatic effects. Moreover, the absence of variability within accessions based on both morphological and molecular markers allowed us to dismiss the possibility of errors occurring during the processes of propagation and plantation of accession trees (Tables 1 and 2; Supplemental Table 1).

Molecular similarities among different genotypes were evaluated using the Dice's similarity index. A wide range of similarity values was found between all possible pairs of accessions (data not shown), ranging from 0.645 to 1 . A dendrogram was constructed by unweighted pair group method with arithmetic mean algorithm (Fig. 2). The cophenetic correlation coefficient between the dendrogram and the original similarity matrix was significant and high $(\mathrm{r}=0.99 ; P<0.01)$, which indicates a good fit of the original data to the clustering. According to cluster analysis, the accessions were classified into three groups (I, II, and III) sharing a similarity index from 0.645 to 0.650 among them. Group I included five accessions: Kiti, Klirou 2, Kato Drys, and Kato Drys 1 from Nicosia and Larnaca region and one accession of unknown origin. This set of accessions shared high similarity indexes $\geq 0.9$ among them. The pair of accessions Kiti-Unknown shared identical SSR profile (Profile 1). Group II was the most important in terms of the number of genotypes (11) and accessions (26), of which $22(85 \%)$ and four (15\%) were collected in the Nicosia and Larnaca districts, respectively (Table 1). The similarity indexes among these accessions also were high, ranging from 0.915 to 1 . Seven of the accessions belonging to this group showed unique SSR profiles $(7,8,9,10,11,13$, and $15)$ and the remaining 19 accessions shared their SSR profiles with other accessions within Group II, resulting in the identification of the remaining four SSR profiles $(5,6,12$, and 14; Tables 1 and 2). SSR profile 5 was the most abundant in the collection, with 12 accessions sharing this profile, which may support the case that it was a primary genotype. The Korakou accession was grouped independently to Groups I and II, with which it shared low values of similarity $(0.64-0.65)$.

We observed that differences among genotypes within each group were always associated with minor differences (one or two alleles) detected in only one locus and corresponding at the same time to a difference of $1 \mathrm{bp}$ between alleles (Table 2). The consistency of these profiles was confirmed by reamplification of the samples. Up to seven (ssrOeUA-DCA3, ssrOeUA-DCA9, ssrOeUADCA11, ssrOeUA-DCA16, ssrOeUA-DCA18, GAPU103A, and UDO99-043) of 12 polymorphic SSRs were involved in the amplification of these small allelic differences (Table 4). Such variations in DNA have been reported in olive (Anestiadou et al., 2017; Chalak et al., 2015; Charafi et al., 2008; Díez et al., 2011; Muzzalupo et al., 2010; Trujillo et al., 2014) as well as in other species, such as the grapevine, which commands a pattern of culture very similar to the olive concerning its diffusion, history, propagation system, and varietal diversity (Riaz et al., 2002; This et al., 2006). These variations could be attributed to somatic mutations caused by the sequential clonal propagation of traditional varieties as well as to the type of molecular marker used, especially microsatellite types, as somatic mutations are 


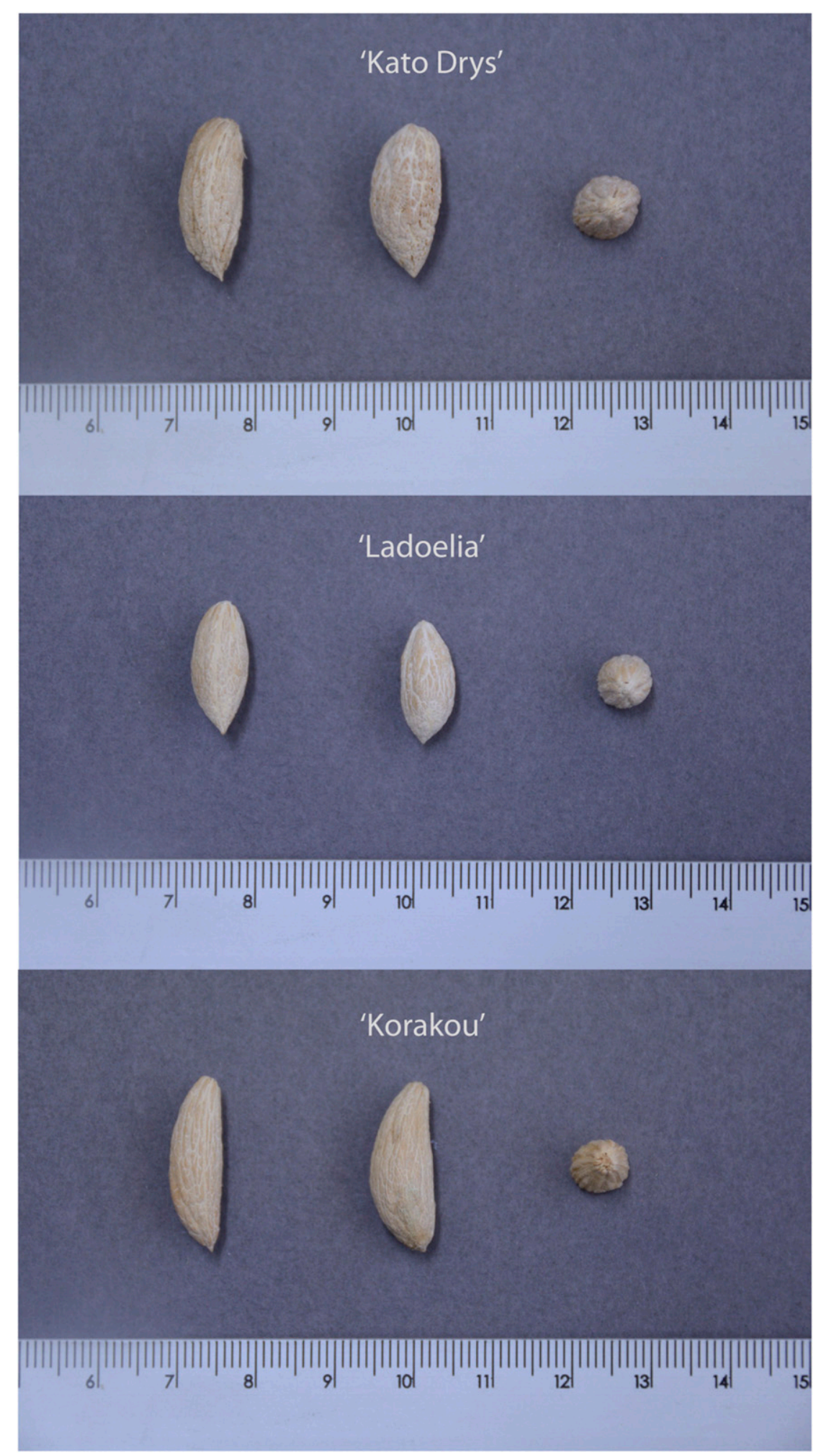

Fig. 1. Position A, B, and C (as described in International Union for the Protection of New Varieties of Plants TG/99/4) of endocarps of the three cultivars identified in the current study: 'Kato Drys', 'Ladoelia', and 'Korakou'.

more likely to occur in genomic regions highly variable and neutral evolving like the SSRs (Díez et al., 2011; Trujillo et al., 2014).

Cataloguing of the collection: identification and naming of cultivars. The use of OCARICY as the reference genetic collection in the

HortScience Vol. 53(9) September 2018 context of the recently developed National Register of Commercial Varieties in Cyprus requires its appropriate cataloguing. For this purpose, we have used the criteria described in the protocol by Trujillo et al. (2014) for cataloguing the genetic resources preserved in olive germplasm banks, which is based on the integrated use of morphological and molecular markers (microsatellites). Thus, the category "cultivar" was assigned to accessions that presented morphological and/or molecular distinctness, whereas the category "molecular variants" was considered for those accessions that presented minimum variance in their molecular profiles $(\mathrm{SI} \geq 0.9$ ) but their morphological profiles were identical and referred to the same cultivar. Previous studies relied unilaterally on morphological (Gregoriou, 1996, 1999) or molecular characterization of the Cyprus olive germplasm and interpreted the variability observed among 'Ladoelia' accessions in the context of a multiclonal cultivar (Anestiadou et al., 2017; Banilas et al., 2003). By contrast, the present work constitutes the first characterization study on the entire OCARICY collection by combining SSR and morphological profiles. This methodology has enabled the identification and first report of the presence of three cultivars and 15 molecular variants among the 32 accessions of the OCARICY collection (Table 1).

The morphological grouping of accessions per cultivar was in agreement with that obtained by SSR markers. In other words, all accessions clustered in the same group according to their SSR profiles also demonstrated identical morphological profiles. For example, the five accessions clustered into Group I by SSR markers with similarity indexes $\geq 0.9$ presented the same morphological profile (Phenotype 1) and belonged to the same cultivar (Fig. 2; Table 1). The second cultivar (Group II) included 26 accessions that shared morphological profile 2 (Phenotype 2) and 11 similar molecular profiles (SI $\geq 0.9$ ) (Fig. 2). The third cultivar (Group III), labeled as Korakou, exhibited singular molecular and morphological profiles, numbered 16 and 3 , respectively. This cultivar was the most distinct of the cultivars identified in the OCARICY collection (Figs. 1 and 2; Tables 2 and 3). These results are not far from what we expected, since the germplasm under study comes from a clonal selection of 'Ladoelia', which renders perfectly reasonable the fact that $81 \%$ of the accessions corresponded to this cultivar. The grouping of accessions based on combined morphological and molecular traits has revealed no pattern of geographical clustering (Fig. 2; Tables 1 and 3). For instance, the four accessions (Kiti, Kato Drys, Kato Drys 1, and Klirou 2) presently grouped under the first cultivar includes accessions originated by Larnaca and Nicosia county and even from distant regions. By combining the study of morphological and molecular profiles, we verified the value of this methodology for conducting similar studies on olive germplasm identification. Moreover, we have demonstrated that microsatellite markers UDO99-024 and GAPU 59 were each one in itself capable of discriminating the three identified cultivars of OCARICY without further discriminating intracultivar variability, hence their use is proposed for the efficient identification of olive genetic 


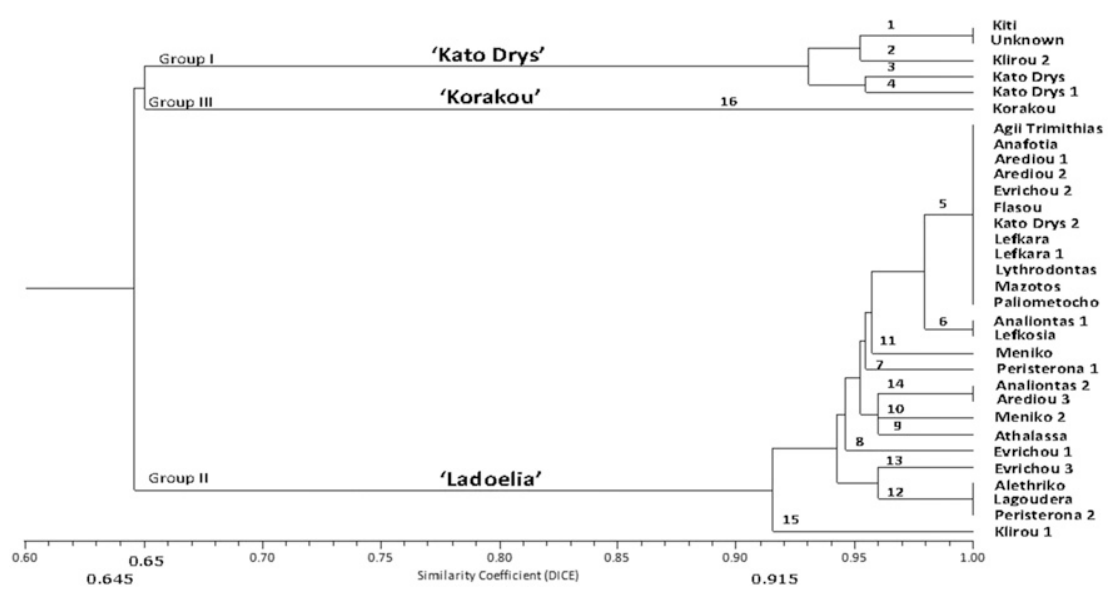

Fig. 2. Unweighted pair group method with arithmetic mean dendrogram based on the Dice similarity index of the 39 trees (duplications are not shown) of the 32 accessions of the Olive Collection of the Agricultural Research Institute of Cyprus depicting the clustering of three identified varieties, based on SSR markers. Numbers 1-15 indicate molecular variants of each variety nominally specified.

material with reference to the National Register of Commercial Varieties of Cyprus.

Similar grouping of accessions to the present (Fig. 2; Supplemental Table 1) was reported in previous agronomic and morphological studies conducted with reference to the OCARICY collection. For instance, the four accessions (Kiti, Kato Drys, Kato Drys 1 , and Klirou 2) presently grouped under the first cultivar had been noted for their greater fruit size (5.5-6.4 g) compared with the accessions of second cultivar $(2.8-3.8 \mathrm{~g})$ in the studies by Gregoriou (1996, 1999), who also reported distinct oil contents in the accessions of the first $(18.9 \%-21.8 \%)$, the second $(25.5 \%-37.2 \%)$, and third cultivar $(13.9 \%)$. It is possible that the wide range of oil content reported for the second cultivar derived partly from harvest maturity differences during the sampling of the various accessions. Although pronounced cases of distinctness among the OCARICY accessions had been reported in the aforementioned studies by Gregoriou (1996, 1999), the number of morphological variables characterized were limited; hence, no identification of distinct cultivars was proposed. The integrated use of morphological and molecular SSR markers adopted in the present study has allowed the identification of three distinct cultivars and 15 molecular variants within the OCARICY collection, with limited molecular and absent morphological variability between accessions of the same cultivar. The existence of molecular variants is not surprising, as it has been reported similarly in traditional varieties from other countries in which genetic diversity may accumulate via mutation events in clonally propagated centennial olives (Chalak et al., 2015; Charafi et al., 2008; Díez et al., 2011; D'Imperio et al., 2011; Khadari et al., 2008; Muzzalupo et al., 2010; Soleri et al., 2010; Trujillo et al., 2014).

Trujillo et al. (2014) previously have described a small number of Cypriot genotypes now included in the WOGBC database. Comparing the morphological and molecular profiles for the OCARICY accessions reported in the present study with those of the entire WOGBC database, we found that the first and third cultivar of the OCARICY do not match any of the non-Cypriot genetic material included in WOGBC database. The first cultivar (represented by five accessions) showed identical morphological profiles and identical or nearly identical SSR profiles $(\mathrm{SI} \geq 0.9)$ to the WOGBC database cv. Kato Drys from Cyprus identified by Trujillo et al. (2014). The third cultivar is represented in the OCARICY collection by a single accession for which no information was available other than its place of origin, Korakou village; hence, the name assigned to this accession in the OCARICY collection (Korakou) was adopted as the cultivar name in the current study.

The results currently obtained and crossreferenced with the WOGBC database allowed us to assign the correct varietal name to each accession of the OCARICY collection (Table 1), as well as to identify new cases of synonymy (different names for the same cultivar used in different growing areas) among the OCARICY collection material. Of particular interest is the second cultivar presently described, which matches not only the profiles of Cypriot accessions included in the WOGBC but additionally that of cv. Beladi from Lebanon. It is thus apparent that the names Ladoelia or Ntopia and Beladi are used respectively for the same cultivar in Cyprus and Lebanon, whereas both cultivar names (i.e., Ntopia in Greek and Beladi in Arabic) designate this cultivar as "local." Notwithstanding the aforementioned synonymy, 'Ladoelia' is the cultivar name registered in the World Catalogue of Olive Varieties, with Cyprus being the assigned cultivar's putative area of origin (International Olive Oil Council, 2000). The case of synonymy between the varieties 'Ladoelia' and 'Beladi' should not surprise us, taking into account the geographical proximity between the two respective countries of putative origin, Cyprus and Lebanon. Atienza et al. (2013) have also reported the affinity of the Cypriot accession Athalassa included in WOGBC to cv. Beladi (or Souri) by comparing their Diversity Arrays Technology (DArT) profiles. Chalak et al. (2015) have actually termed 'Beladi' as a landrace characterized by multiple molecular variants attributed to somaclonal mutations. Our present findings also highlight the high intracultivar molecular variability encountered in synonym 'Ladoelia' associated with minor differences detected in only one locus and corresponding at the same time to a difference of 1 bp between alleles, confirming that the intracultivar molecular variability emerged from somaclonal mutations. By exploiting the SSR profiles of the WOGB Marrakech produced by Haouane et al. (2011), Chalak et al. (2015) have also demonstrated the affinity of 'Beladi' with olive genetic material from Cyprus, attesting thereby to the ancient exchanges of olive germplasm between Lebanon and Cyprus. The aforementioned findings undoubtedly constitute a significant contribution to the unraveling of the diffusion events pertaining to cultivated olive in the Eastern Mediterranean.

\section{Conclusions}

The implementation of the National Register of Commercial Varieties and the use of the OCARICY as reference collection for the olive genetic material of Cyprus warranted prior characterization and cataloguing based on robust discriminative and contrasted methodologies (molecular vs. morphological) developed for olive. Integrated use of morphological and molecular markers for cataloguing the OCARICY accessions has facilitated the detection of synonymies and the identification of distinct cultivars. The use of SSR markers for the discrimination of intravarietal molecular variants provided access to a source of genetic variability previously ignored but potentially important for further evaluation of agronomic characters of interest. Our results further highlight the importance of conducting the identification process along with clonal prospection to reveal the presence of distinct varietal material. We have demonstrated that the OCARICY genetic material belongs to three distinct cultivars and thus repudiated the assumption underlying the original prospection and subsequent studies on this material that considered the OCARICY as a multiclonal collection derived from a single cultivar. The current findings corroborate recent molecular characterization of centennial olive trees of Cyprus in raising the question whether further unexplored olive genetic material exists in Cyprus requiring characterization and conservation.

\section{Literature Cited}

Anestiadou, K., N. Nikoloudakis, M. Hagidimitriou, and A. Katsiotis. 2017. Monumental olive trees of Cyprus contributed to the establishment of the contemporary olive germplasm. PLoS One 12(11): E0187697, doi: 10.1371/journal.pone.0187697. 
Atienza, S.G., R. de la Rosa, M.C. DomínguezGarcía, A. Martín, A. Kilian, and A. Belaj. 2013. Use of DArT markers as a means of better management of the diversity of olive cultivars. Food Res. Intl. 54:2024-2053.

Baldoni, L., N.G. Cultrera, R. Mariotti, C. Ricciolini, S. Arcioni, G. Vendramin, and R. Testolin. 2009. A consensus list of microsatellite markers for olive genotyping. Mol. Breed. 24(3):213-231.

Banilas, G., J. Minas, C. Gregoriou, C. Demoliou, A. Kourti, and P. Hatzopoulos. 2003. Genetic diversity among accessions of an ancient olive variety of Cyprus. Genome 46:370-376.

Barranco, D., A. Cimato, P. Fiorino, L. Rallo, A. Touzani, C. Castañeda, F. Serafín, and I. Trujillo. 2000. World catalogue of olive varieties. International Olive Oil Council, Madrid.

Barranco, D., I. Trujillo, and L. Rallo. 2005. Libro I. Elaiografía Hispánica, p. 52-58. In: L. Rallo, D. Barranco, J.M. Caballero, C. Del Rio, A. Martin, J. Tous, and I. Trujillo (eds.). Variedades de olivo en España. Junta de Andalucía, MAPA y Ediciones Mundi-Prensa. Madrid.

Bartolini, G., G. Prevost, C. Messeri, and C. Carignani. 1998. Olive germplasm: Cultivars and world-wide collections. Seed and Plant Genetic Resources Service, Food and Agriculture Organization of the United Nations, Rome.

Besnard, G., B. Khadari, M. Navascués, M. Fernández-Mazuecos, A. El Bakkali, N. Arrigo, D. Baali-Cherif, V. Brunnini-Bronzini de Caraffa, S. Santoni, P. Vargas, and V. Savolainen. 2013. The complex history of the olive tree: From Late Quaternary diversification of Mediterranean lineages to primary domestication in the northern Levant. Proc. Biol. Sci. 280:20122833.

Botstein, D., R.L. White, M. Skolnick, and R.W. Davis. 1980. Construction of a genetic linkage map in man using restriction fragment length polymorphisms. Amer. J. Hum. Genet. 32:314-331.

Brickell, C.D., C. Alexander, J.C. David, W.L.A. Hetterscheid, A.C. Leslie, V. Malecot, J. Xioban, and J.J. Cubey. 2009. International Code of Nomenclature for Cultivated Plants, Scripta Horticulturae. 8th ed. ISHS, 10, Vienna.

Carriero, F., G. Fontanazza, F. Cellini, and G. Giorio. 2002. Identification of simple sequence repeats (SSRs) in olive (Olea europaea L.). Theor. Appl. Genet. 104:301-307.

Charafi, J., A. El Meziane, A. Moukhli, B. Boulouha, C. El Modafar, and B. Khadari. 2008. Menara gardens: A Moroccan olive germplasm collection identified by a SSR locus-based genetic study. Genet. Resources Crop Evol. 55:893-900.

Chalak, L., H. Haouane, L. Essalouh, S. Santoni, G. Besnard, and B. Khadari. 2015. Extent of the genetic diversity in Lebanese olive (Olea europaea L.) trees: A mixture of an ancient germplasm with recently introduced varieties. Genet. Resources Crop Evol. 62:621-633.

Cipriani, G., M.T. Marrazzo, R. Marconi, A. Cimato, and R. Testolin. 2002. Microsatellite markers isolated in olive (Olea europaea L.) are suitable for individual fingerprinting and reveal polymorphism within ancient cultivars. Theor. Appl. Genet. 104(2-3):223-228.

D’Imperio, M., V. Viscosi, M. Scarano, M. D'Andrea, B. Zullo, and F. Pilla. 2011. Integration between molecular and morphological markers for the exploitation of olive germplasm (Olea europaea). Scientia Hort. 130:229-240.

De La Rosa, R., C.M. James, and K.R. Tobutt. 2002. Isolation and characterization of polymorphic microsatellites in olive (Olea europaea L.) and their transferability to other genera in the oleaceae. Mol. Ecol. Notes 2(3):265-267.

Dice, L.R. 1945. Measures of the amount of ecologic association between species. Ecology 26:297-302.

Díez, C.M., A. Imperato, L. Rallo, D. Barranco, and I. Trujillo. 2012. Worldwide core collection of olive cultivars based on simple sequence repeat and morphological markers. Crop Sci. 52:211-221.

Díez, C.M., I. Trujillo, E. Barrio, A. Belaj, D. Barranco, and L. Rallo. 2011. Centennial olive trees as a reservoir of genetic diversity. Ann. Bot. 108:797-807.

Fendri, M., I. Trujillo, A. Trigui, I.M. RodriguezGarcia, and J. De Dios Alche Ramirez. 2010 Simple sequence repeat identification and endocarp characterization of olive tree accessions in a Tunisian germplasm collection. HortScience 45:1429-1436.

Gregoriou, C. 1996. Assessment of variation of landraces of olive tree in Cyprus. Euphytica $87: 173-176$

Gregoriou, C. 1999. Clonal selection of "Local" olive variety of Cyprus. Olivae 76:26-30.

Hadjisavvas, S. 1992. Olive Oil Processing in Cyprus. From the Bronze Age to the Byzantine Period. SIMA, Vol. XCIX. Paul Astroms Forlag, Uppsala.

Haouane, H., A. El Bakkali, A. Moukhli, C. Tollon, S. Santoni, A. Oukabli, C. El Modafar, and B. Khadari. 2011. Genetic structure and core collection of the World Olive Germplasm Bank of Marrakech: Towards the optimised management and use of Mediterranean olive genetic resources. Genetica 139(9):1083-1094.

Khadari, B., J. Charafi, A. Moukhli, and M. Ater. 2008. Substantial genetic diversity in cultivated Moroccan olive despite a single major cultivar: A paradoxical situation evidenced by the use of SSR loci. Tree Genet. Genomes 4:213-221.

Lavee, S. 1994. ¿Por qué la necesidad de nuevas variedades de olivos? Fruticultura 62:29-37.

Liu, K.J. and S.V. Muse. 2005. Power Marker: An integrated analysis environment for genetic marker analysis. Bioinformatics 21:21282129.

Murray, M.G. and W.F. Thompson. 1980. Rapid isolation of high molecular weight plant DNA. Nucleic Acids Res. 8:4321-4326.
Muzzalupo, I., A. Chiappetta, C. Benincasa, and E. Perri. 2010. Intra-cultivar variability of three major olive cultivars grown in different areas of central-southern Italy and studied using microsatellite markers. Scientia Hort. 126:324-329.

Paetkau, D. and C. Strobeck. 1994. Microsatellite analysis of genetic-variation in black bear populations. Mol. Ecol. 3:489-495.

Rafalski, J.A. and S.V. Tingey. 1993. Genetic diagnostics in plant breeding: RAPDs, microsatellites and machines. Trends Genet. 9:275280.

Rallo, L. 2005. Variedades de olivo en España: Una aproximación cronológica, p. 17-44. In: Variedades de olivo en España. L. Rallo, D. Barranco, J.M. Caballero, C. Del Río, A. Martín, J. Tous, and I. Trujillo (eds.). Junta de Andalucía, MAPA y Ediciones Mundi-Prensa. Madrid.

Rallo, P., G. Dorado, and A. Martín. 2000. Development of simple sequence repeats in olive tree (Olea europaea L.). Theor. Appl. Genet. 101:984-989.

Riaz, S., K.E. Garrison, and G.S. Dangl. 2002. Genetic divergence and chimerism within ancient asexually propagated winegrape cultivars. J. Amer. Soc. Hort. Sci. 127:508-514.

Rohlf, F.J. 1998. NTSYS-pc. Numerical taxonomy and multivariate analysis system. Version 2.00. Exeter Software, Setauket, NY

Sefc, K.M., M.S. Lopes, D. Mendonça, M. Rodrigues Dos Santos, M. Laimer Da Câmara Machado, and A. Da Câmara Machado. 2000. Identification of microsatellite loci in olive (Olea europaea) and their characterization in Italian and Iberian olive trees. Mol. Ecol. 9(8): 1171-1173.

Soleri, D., A. Koehmstedt, M.K. Aradhya, V. Polito, and K. Pinney. 2010. Comparing the historic olive trees (Olea europaea L.) of Santa Cruz Island with contemporaneous trees in the Santa Barbara, CA area: A case study of diversity and structure in an introduced agricultural species conserved in situ. Genet. Resources Crop Evol. 57:973-984.

This, P., T. Lacombe, and M.R. Thomas. 2006. Historical origins and genetic diversity of wine grapes. Trends Genet. 22:511-519.

Trujillo, I., A.M. Ojeda, M.N. Urdiroz, D. Potter, D. Barranco, L. Rallo, and M.C. Diez. 2014. Identification of the Worldwide Olive Germplasm Bank of Córdoba (Spain) using SSR and morphological markers. Tree Genet. Genomes 10:141-155.

Trujillo, I., M.A. Ojeda, L. Baldoni, and A. Belaj. 2006. Olive cultivar identification by means of microsatellites (SSR). Olea 25:24-27.

Valière, N. 2002. GIMLET: A computer program for analysing genetic individual identification data. Mol. Ecol. Notes 2(3):377-379.

Zohary, D. and P. Spiegel-Roy. 1975. Beginning of fruit growing in the Old World. Science 187:319-327. 


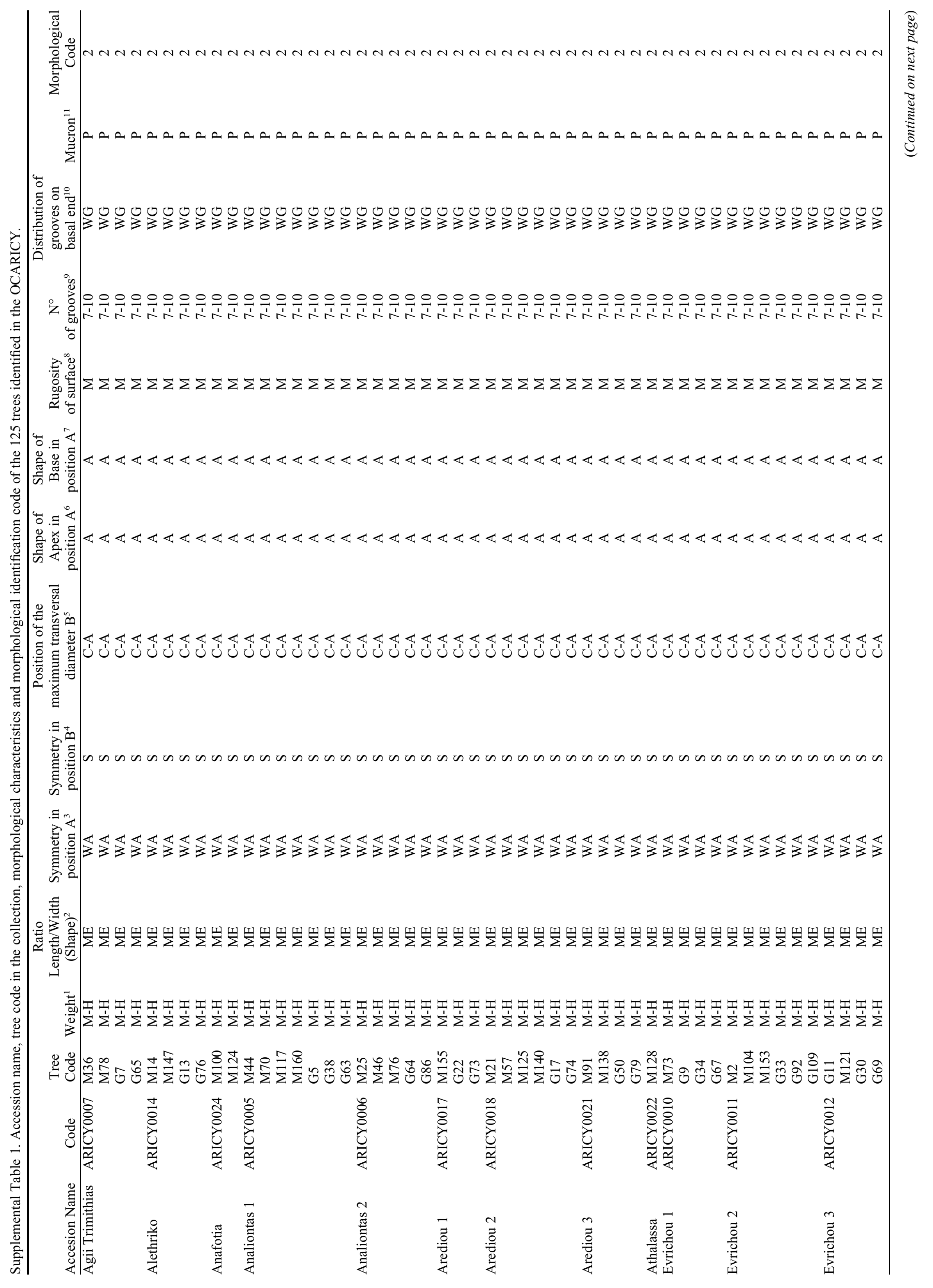

HortScience Vol. 53(9) September 2018 


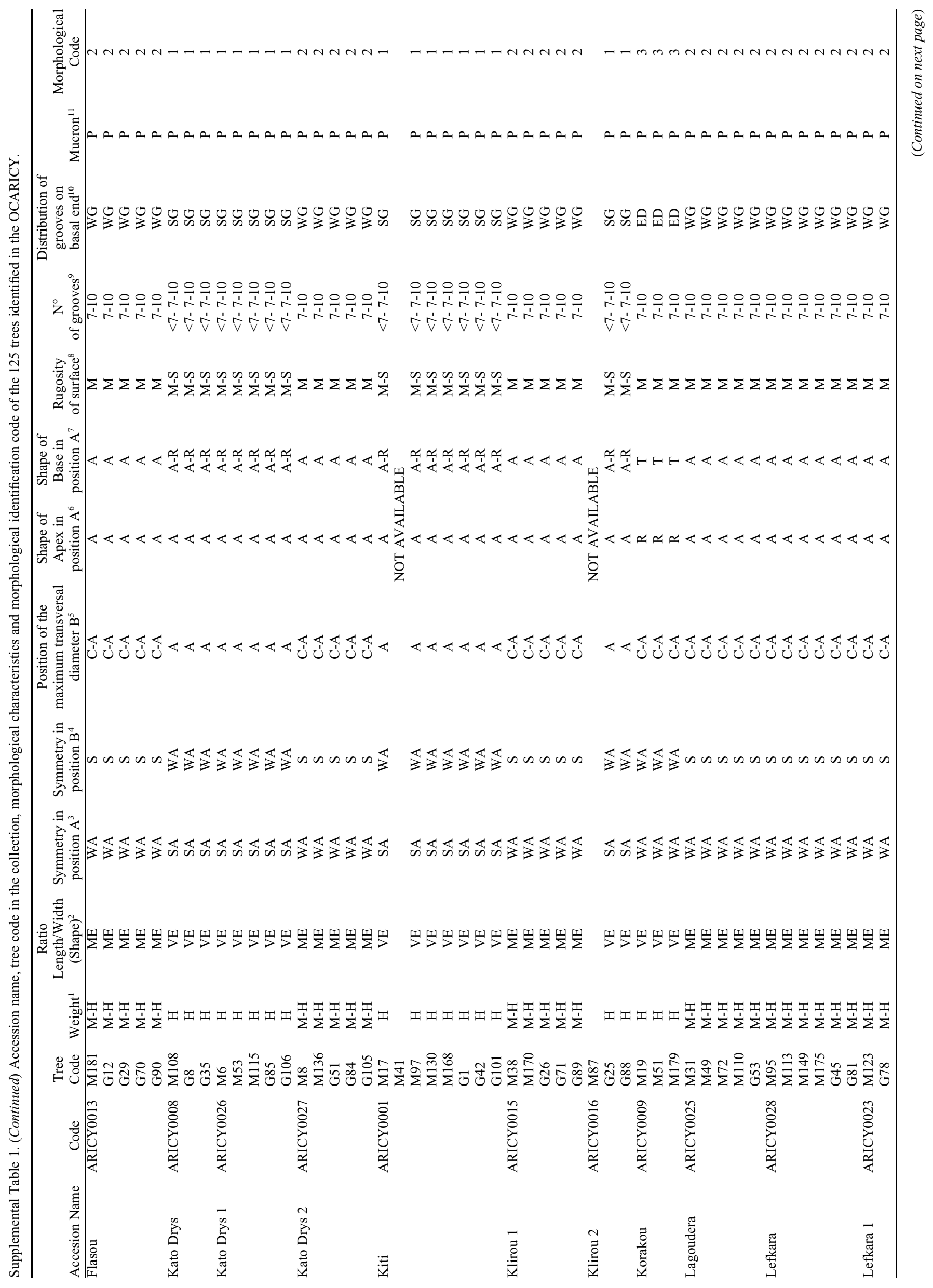




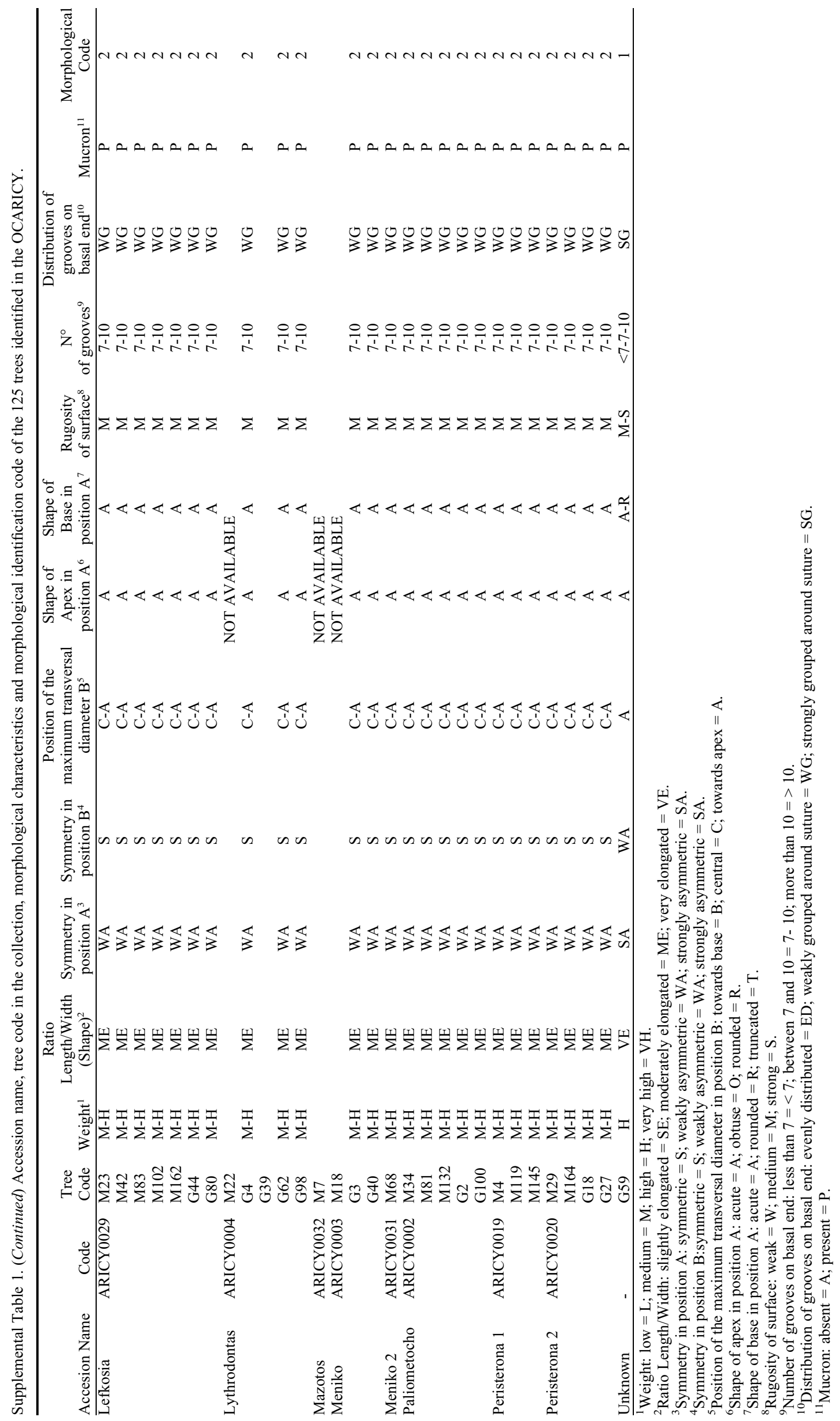

